\title{
Evolution of NV centers in nanodiamond using in situ heating with STEM-EELS/EDS
}

\author{
Bethany Hudak ${ }^{1}$ and Rhonda Stroud ${ }^{2}$ \\ ${ }^{1}$ U.S. Naval Research Laboratory, District of Columbia, United States, ${ }^{2}$ U.S. Naval Research Laboratory, \\ United States
}

Nitrogen-vacancy (NV) centers in diamond are attractive as atomic-scale quantum systems for sensing applications, composed of a nitrogen atom substituted on the carbon lattice with a neighboring vacancy [1]. Advantages of nanodiamond as qubit host material include characteristics such as optical transparency, mechanical hardness, chemical inertness, low magnetic noise, and zero nuclear spin [1]. Since the photoluminescent properties of NV centers are dependent on characteristics including charge state and nanodiamond surface, it is important to be able to fully study the location of a NV center within the diamond lattice [2]. Aberration-corrected scanning transmission electron microscopy (STEM) provides atomic resolution imaging, but a single $\mathrm{N}$ atom lacks the contrast necessary to appear in annular dark field imaging of even a small nanodiamond. Electron energy loss spectroscopy (EELS) can also be used to locate the $\mathrm{N}$ within a nanodiamond, but the weak core-loss signal from a single $\mathrm{N}$ atom at about $400 \mathrm{eV}$ is challenging to resolve. Recent computational literature has shown that an NV center in nanodiamond produces a peak at $282.4 \mathrm{eV}$ near the C-K edge spectrum [3]. Recent work coupling STEM-EELS with energy dispersive Xray spectroscopy (EDS) has experimentally verified this work by simultaneously imaging the $282.4 \mathrm{eV}$ peak in EELS with the $0.392 \mathrm{keV} \mathrm{N}$ peak in EDS, concluding that the $282.4 \mathrm{eV}$ peak does indeed correspond to the presence of a $\mathrm{N}$ atom [4], and previous work has shown that STEM-EDS is capable of single-atom sensitivity [5].

Here we use in situ heating in a NionUltraSTEM-X operated at $60 \mathrm{kV}$ to study the effect of heating nanodiamonds extracted from the Murchison meteorite. Initial results of STEM-EELS/EDS taken from a single nanodiamond before and after heating to $700{ }^{\circ} \mathrm{C}$ (Figure 1) suggest that heating might alter the vacancy electronic state, resulting in the loss of the $282 \mathrm{eV}$ peak, while the $\mathrm{N}$ remains in the diamond lattice. The spectra were acquired by scanning a reduced raster box over a single nanodiamond for $30 \mathrm{~min}$. The EDS data were acquired with a Bruker Xflash 100 windowless detector with an exposure time of 1800 seconds. The EELS data were acquired with a GatanEnfinium ER at $5 \mathrm{sec} /$ frame with a $0.02 \mathrm{eV} / \mathrm{channel}$ dispersion summed over 360 frames. Before and after heating, quantification of the $\mathrm{N}$ content from the EDS spectrum indicates a $\mathrm{N}$-doping level of about $1 \%$. Initially the EELS shows a small peak at $282 \mathrm{eV}$, which disappears after heating. Further investigation is necessary to determine if the NV center undergoes a change in electronic state or if other mechanisms affect the $282 \mathrm{eV}$ peak, such as surface graphitization of the nanodiamond during heating. Another larger nanodiamond with a visible twin boundary (Figure 2) shows no change to the $282 \mathrm{eV}$ peak in EELS or the $\mathrm{N}$ content in EDS upon heating to $700{ }^{\circ} \mathrm{C}$, suggesting that nanodiamond size or the presence of a twin boundary might play a role in the temperature dependence of the NV centers.

Full interpretation of these results is complicated by the unknown formation and processing histories of the meteoritic nanodiamonds. More controlled studies from synthetic nanodiamonds will help us elucidate information such as the NV center preferred location in a nanodiamond and evolution upon heating. Here we show the potential to study the NV centers of nanodiamonds on a single-diamond basis, and by using this technique of simultaneous STEM-EELS/EDS we can further investigate the structure of $\mathrm{NV}$ centers in diamond on an atomistic level and address open questions related to how nanodiamond size and defects impact NV centers [6]. 

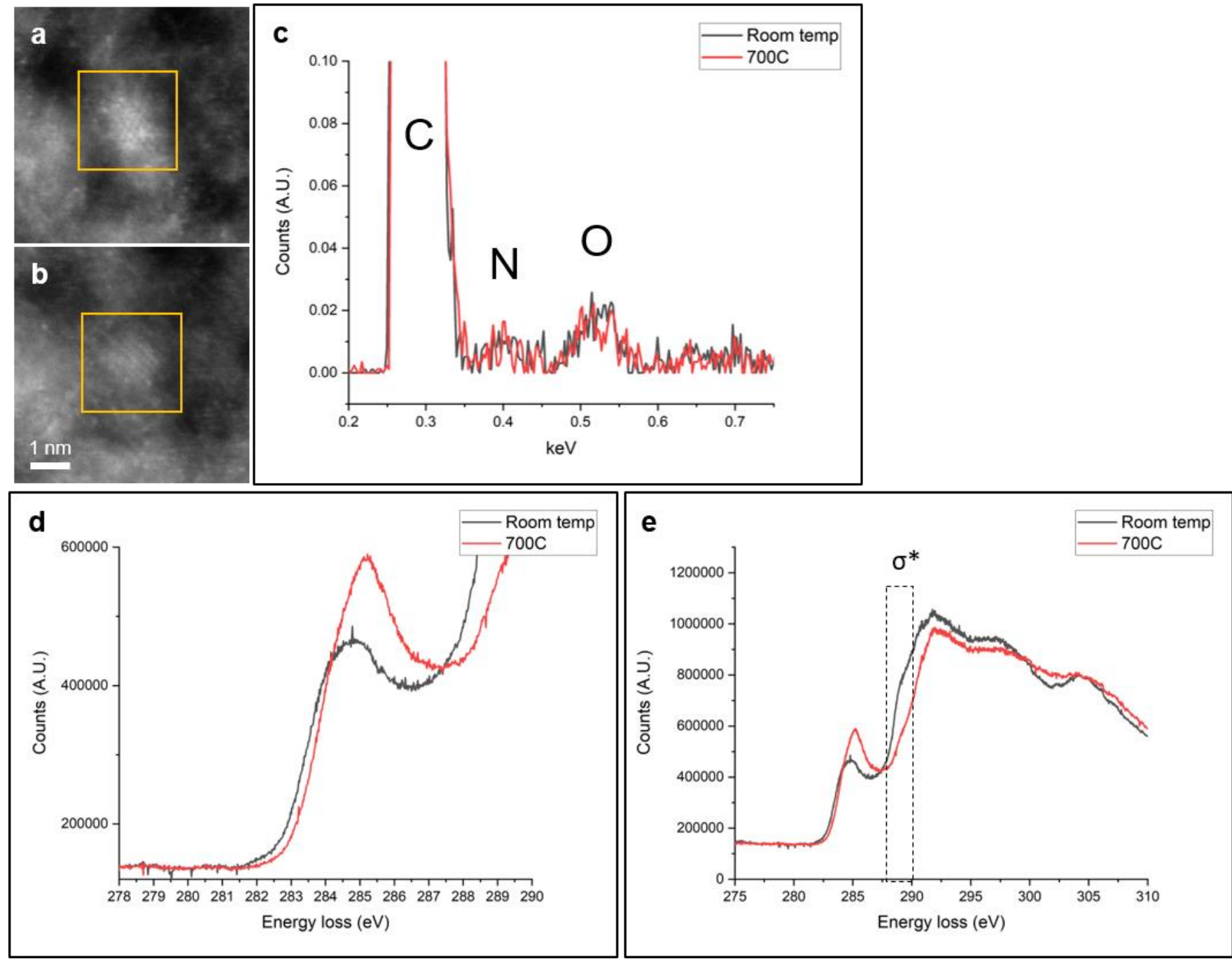

Figure 1. Middle angle annular dark field (MAADF) images of a single nanodiamond (a) before and (b) after heating to $700{ }^{\circ} \mathrm{C}$ with simultaneous (c) EDS and (d-e) EELS. (e) shows the full C-K edge, with the onset of the $\sigma^{*}$ peak highlighted. The loss of the $282 \mathrm{eV}$ peak in EELS and the retained N EDS peak indicate that the NV center charge state has changed upon heating. The shift in the $\sigma^{*}$ onset, the relative intensity of the $\pi^{*}$ at $285 \mathrm{eV}$, and dip at $302 \mathrm{eV}$ indicate that some surface graphitization of the nanodiamonds has also occurred. EELS data was processed to remove large spikes and align the spectra backgrounds. 


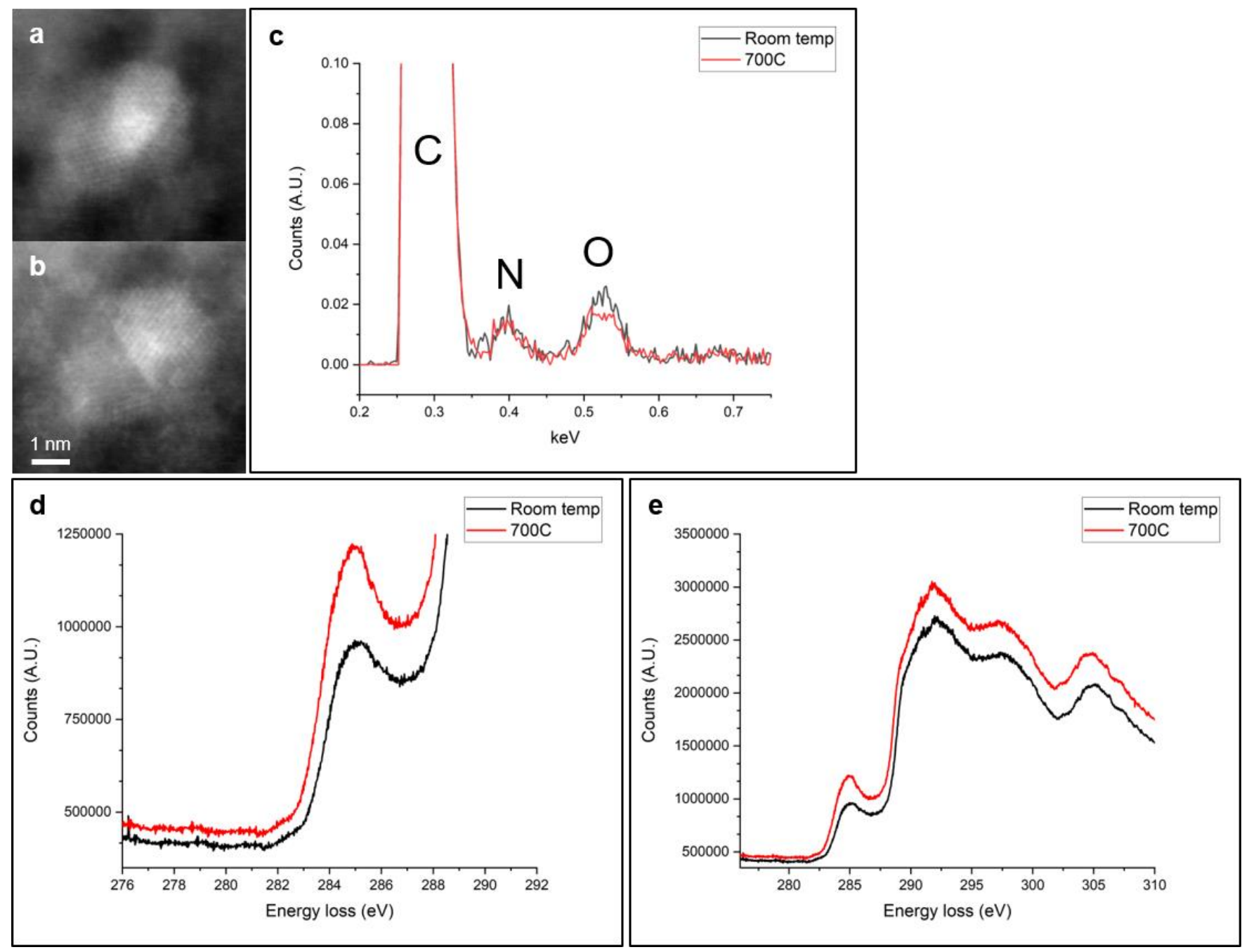

Figure 2. MAADF images of a twinned nanodiamond (a) before and (b) after heating to $700{ }^{\circ} \mathrm{C}$ with simultaneous (c) EDS and (d-e) EELS. The EELS peak at $282 \mathrm{eV}$ is characteristic of the presence of NV centers and does not change upon heating. EELS was processed to remove large spikes.

\section{References}

[1] M. Radtke et al. Nano Futures, 3 (2019), 042004.

[2] C.J. Meara et al. J. Phys. Chem. Solids, 146 (2020), 109637.

[3] S.L.Y. Chang et al. Nanoscale, 8 (2016), 10548.

[4] R.M. Stroud and B.M. Hudak, Microsc. Microanal., 26 (2020), 1506-1507.

[5] R.M. Stroud et al. Appl. Phys. Lett., 108 (2016), 163101.

[6] RMS and BMH acknowledge funding from the NRL 6.1 core program. 